\title{
Historein
}

Vol 8 (2008)

Performing Emotions: Historical and Anthropological Sites of Affect

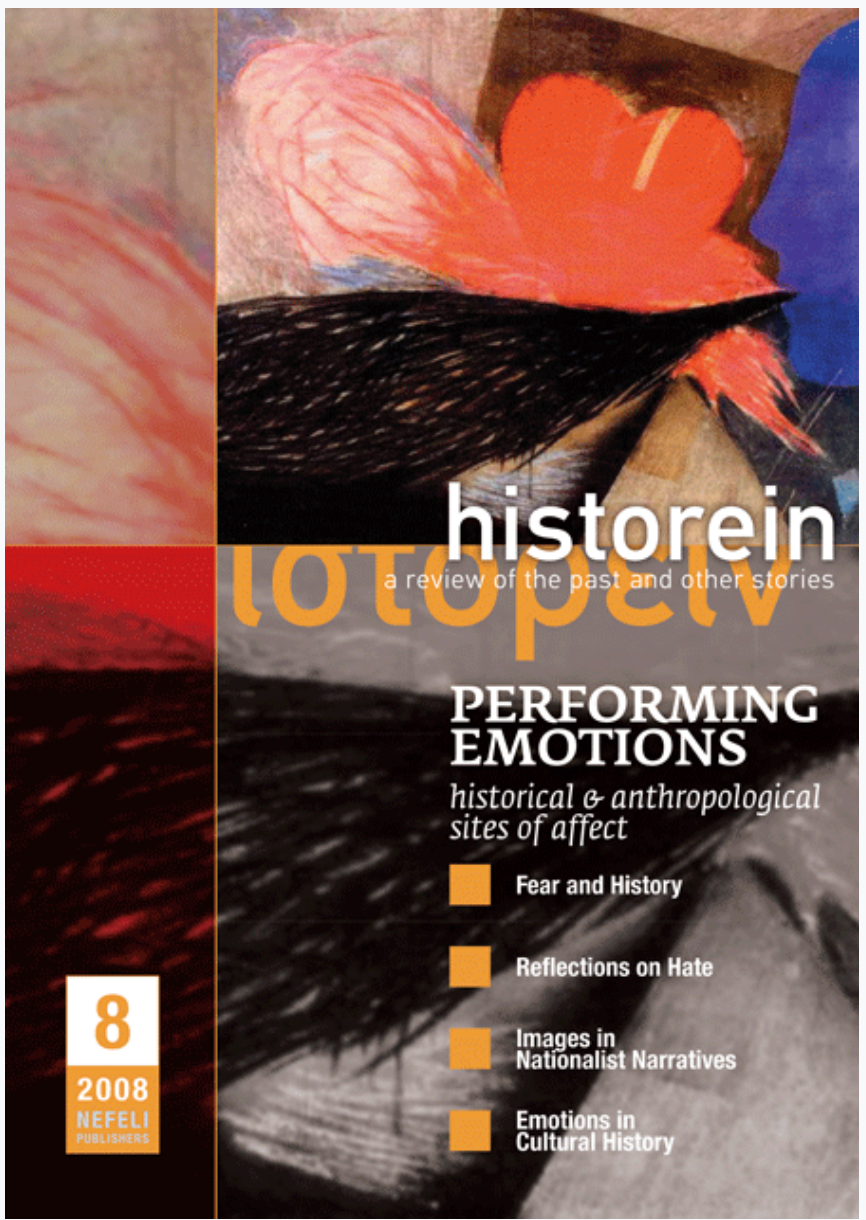

\section{Towards a New Epistemology: The "Affective Turn"}

Athena Athanasiou, Pothiti Hantzaroula, Kostas Yannakopoulos

doi: $10.12681 /$ historein.33

Copyright (C) 2012, Athena Athanasiou, Pothiti Hantzaroula, Kostas Yannakopoulos

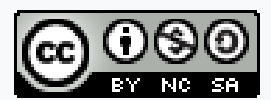

This work is licensed under a Creative Commons Attribution-NonCommercialShareAlike 4.0.

To cite this article:

Athanasiou, A., Hantzaroula, P., \& Yannakopoulos, K. (2009). Towards a New Epistemology: The "Affective Turn". Historein, 8, 5-16. https://doi.org/10.12681/historein.33 


\section{PX B SET B /FX \& QJTUFN PM PHZ 5IFd"G GFDUJWF5V SOj}

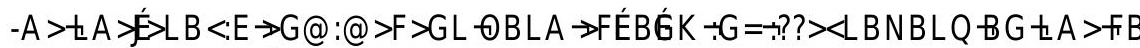

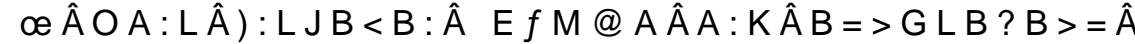

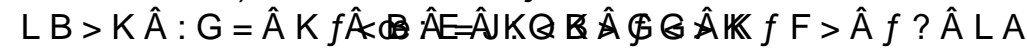

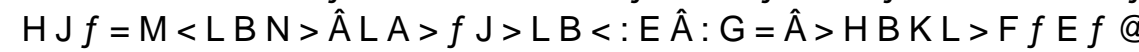

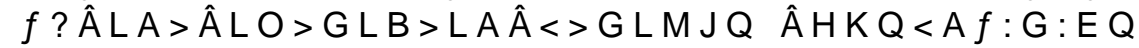

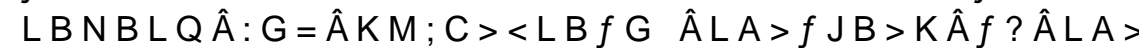

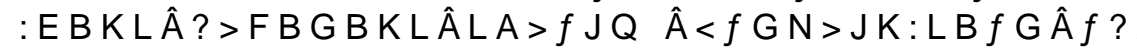

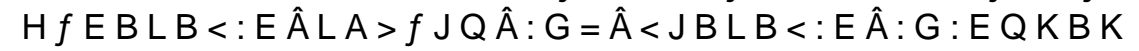

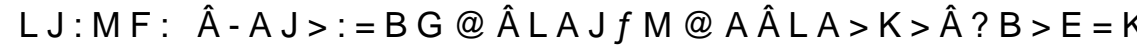

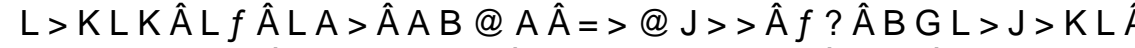

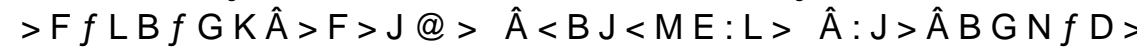

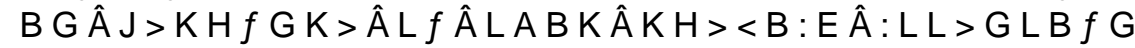

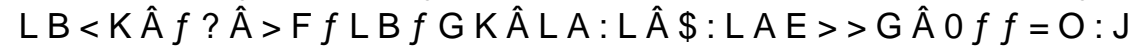

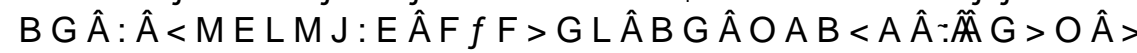

\section{* OUSPEV DUJPC}

$<A E ́$ @́ $B<: E \rightarrow G=B G=B N B=M: E-K L: L>K \rightarrow M L \rightarrow K-K E ́ B: E \rightarrow G=<M A L L M J: E+H J:$

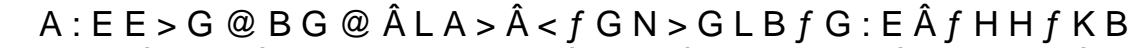

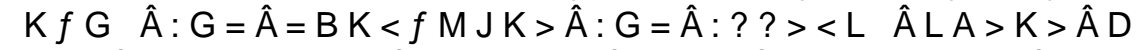

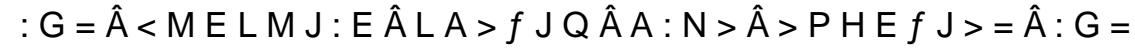


C:OBG@-

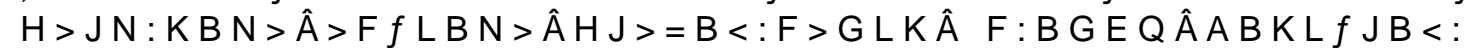

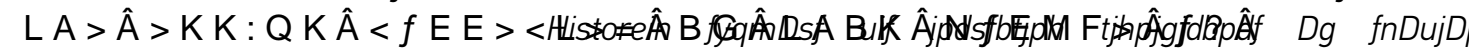
$: G=\rightarrow ? ?><L-B G+F M E L B H E>B G L>J=B K<B H E B G: J Q \rightarrow G=-$ l $: G K=B K<B H E B G: J Q=B K<$ 们 $J K>K \rightarrow$

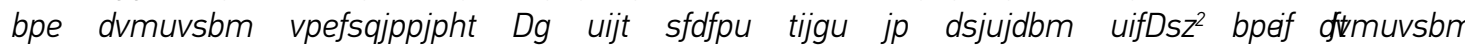
$K: Q K \rightarrow P: F B G>-O A: L\lfloor A B G D B G @ \rightarrow ; \dot{E} L\lfloor-A>-K E ́ B: E B L Q \dot{t} \rightarrow F E ́ B E ́ K \rightarrow G=\rightarrow ? ?><L B N B L Q+>>G$

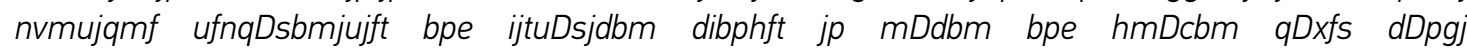

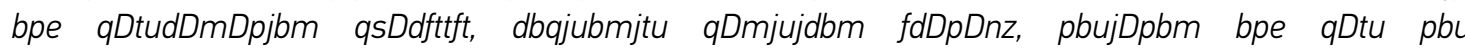

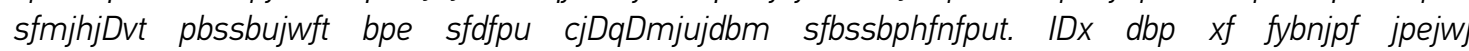

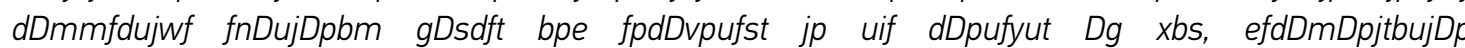

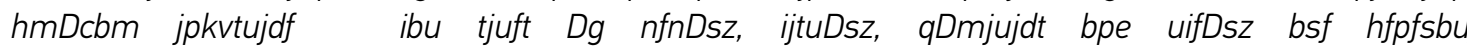

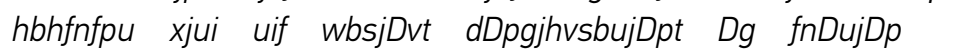

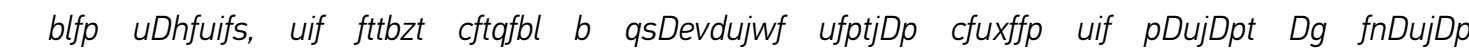

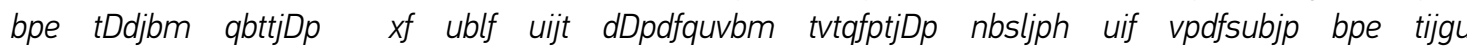

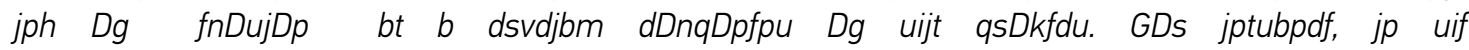

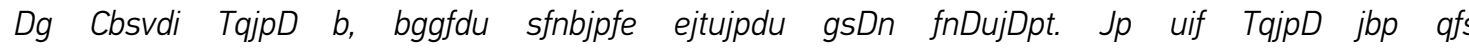

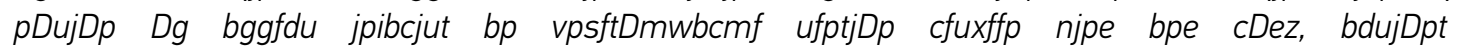

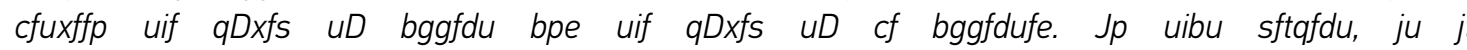

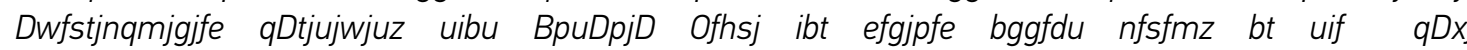

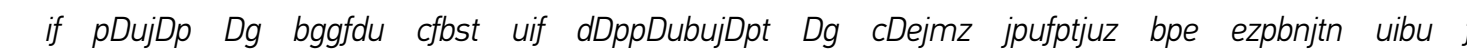

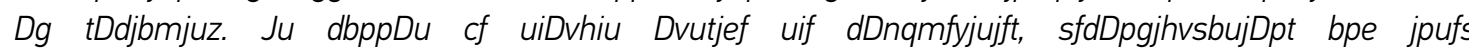

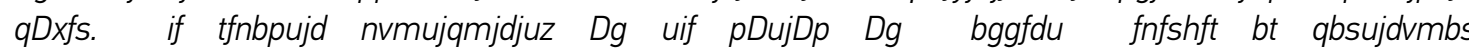

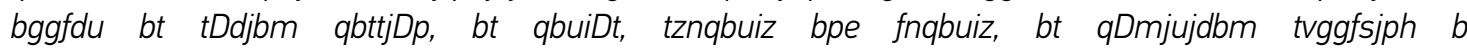

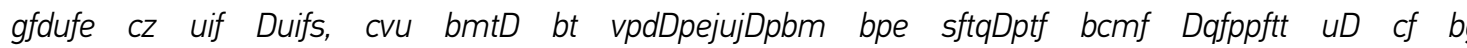

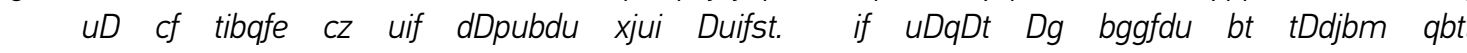

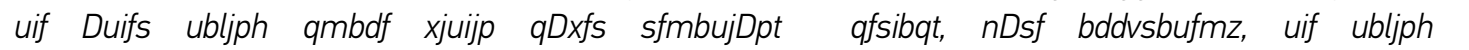

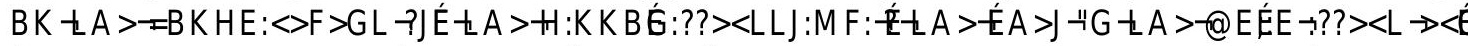

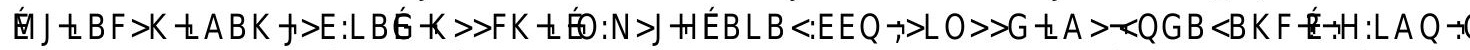

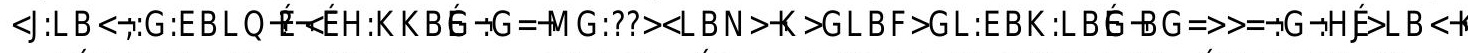

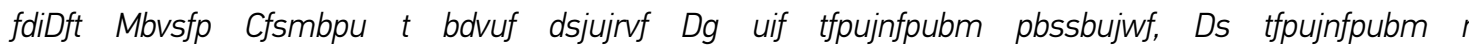

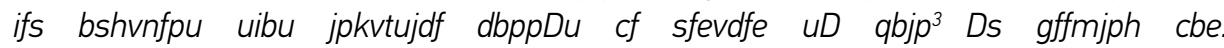

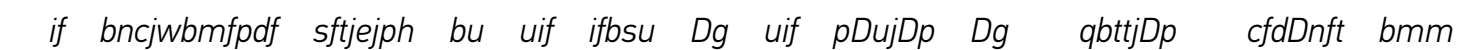

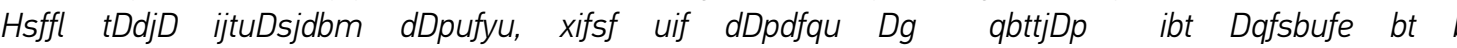

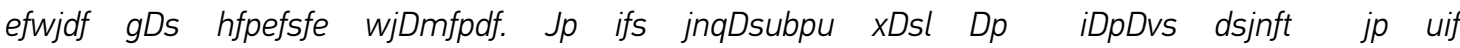

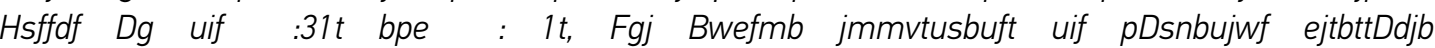

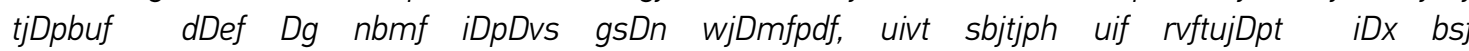

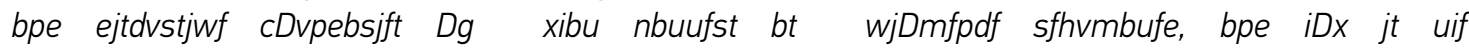

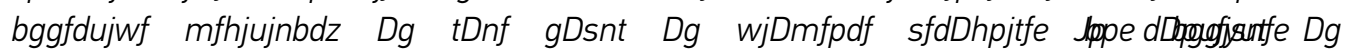

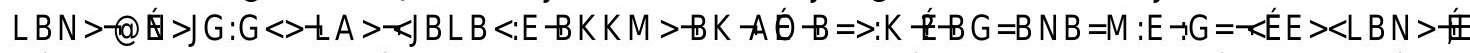

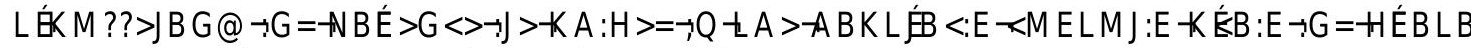

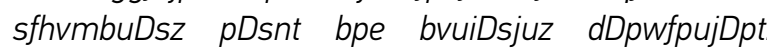




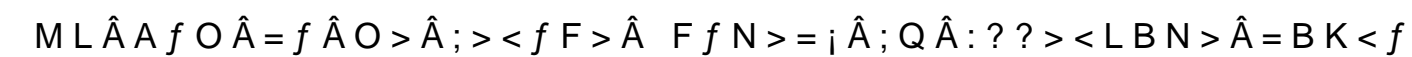

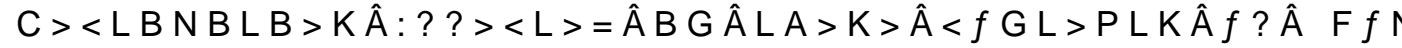

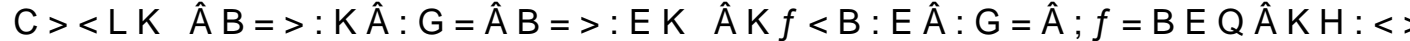

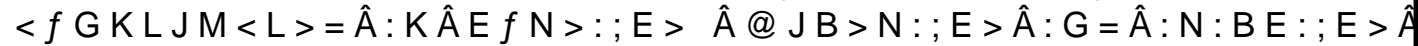

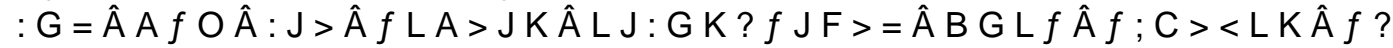

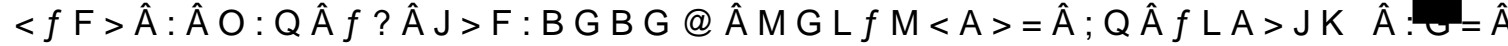

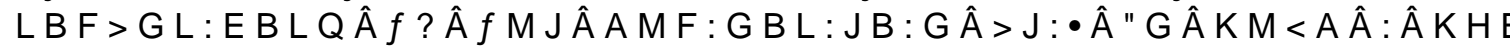

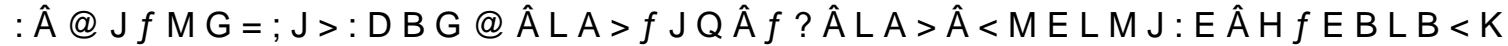

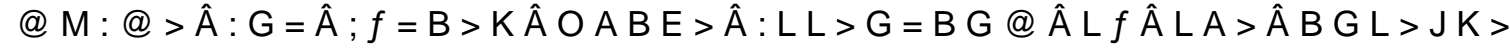

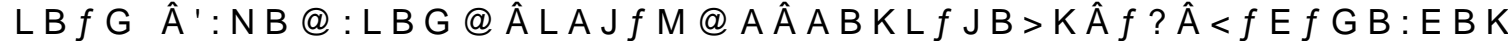

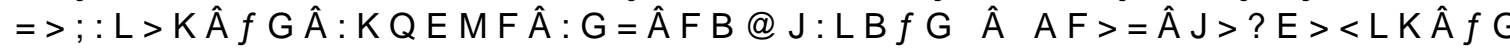
$L A>E ́ Q$

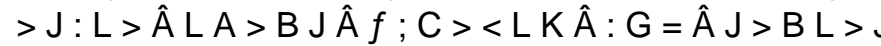

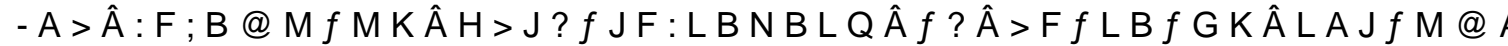

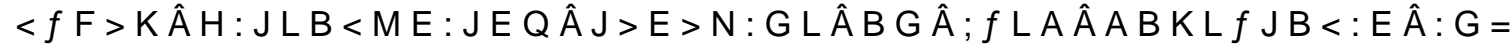
$<: E+H:: L B<>K-B G N E ́ N B G @-K>G L B F>G L: E B L Q<E ́ H: K K B E ́-A M F: G B L: J B: G \rightarrow A>E H \rightarrow G=+H A B E: G$

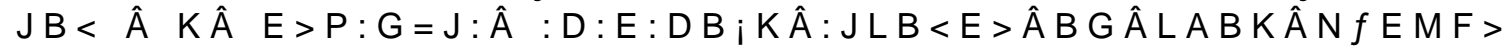

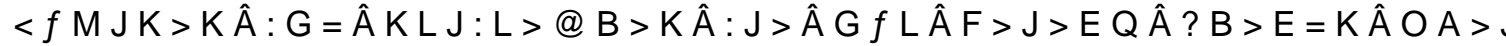

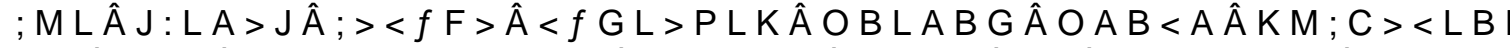

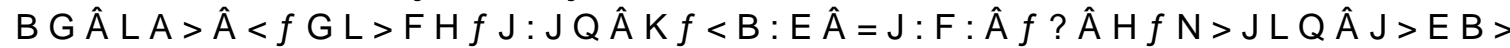

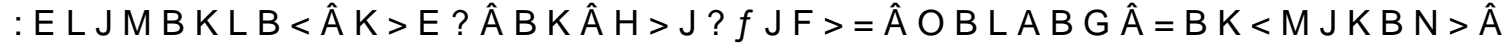

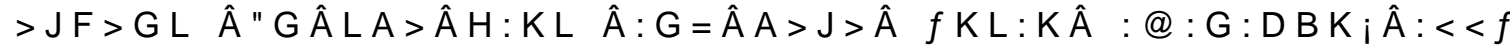

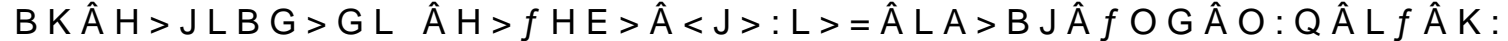

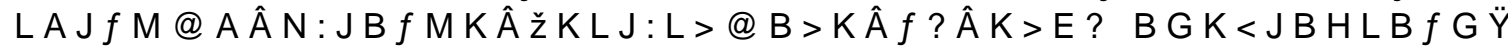

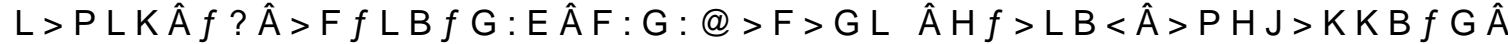

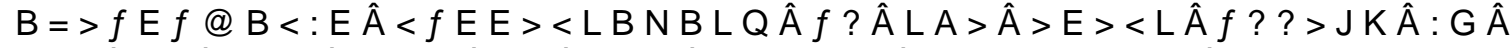

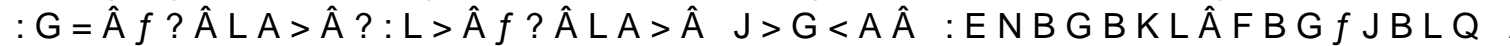

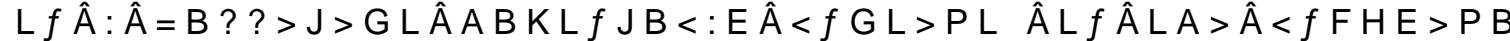

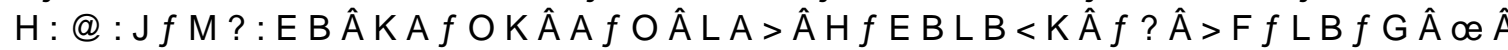

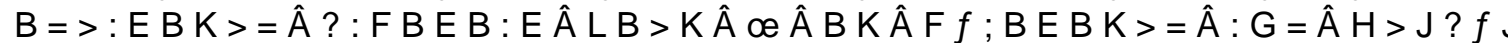

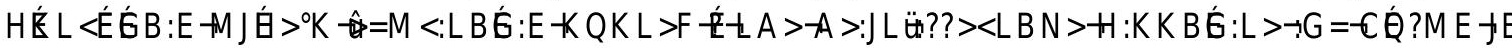

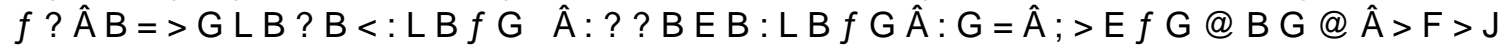

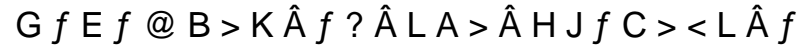

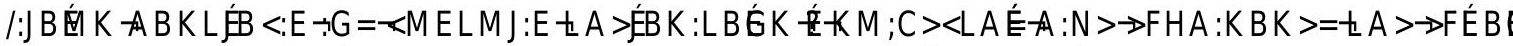
$\Rightarrow>H B G G B G @ K \dot{t}+H \dot{\theta}>J B G+F M E L B H E>-B K<M J K B N>-<\dot{b} L>P L K+\dot{t}-N M E G>1 ; B E B L Q \rightarrow G=-B G C$

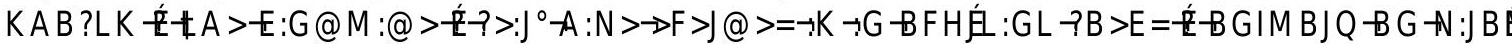

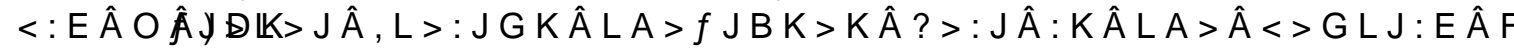

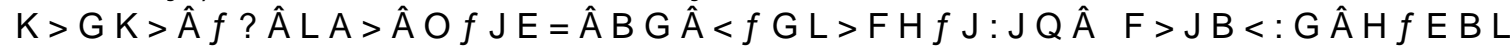

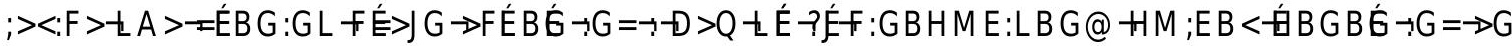

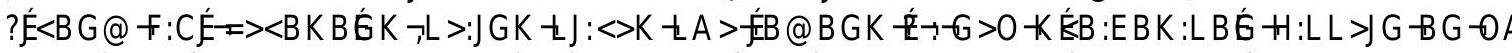

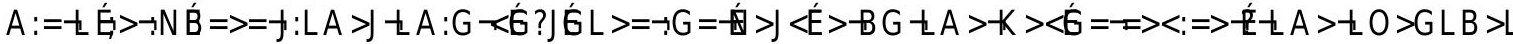

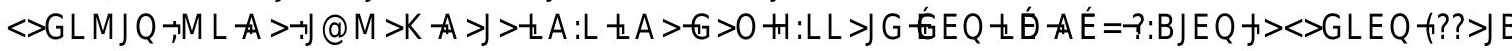




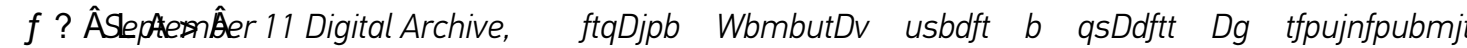

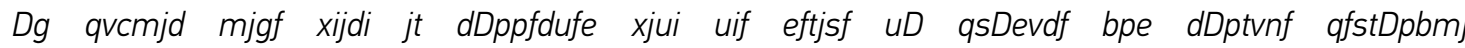

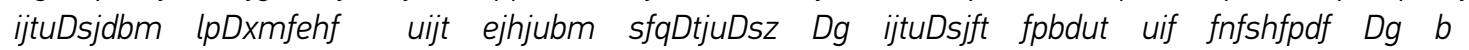

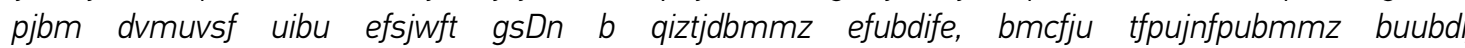

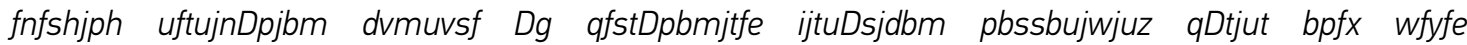

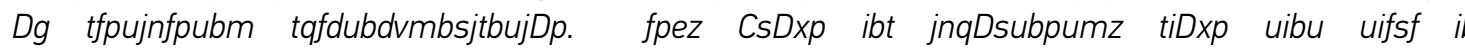

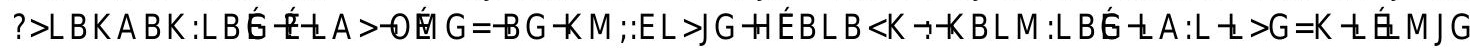

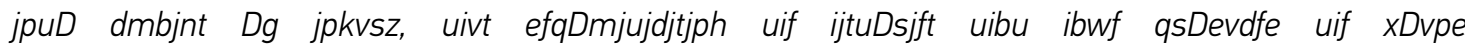

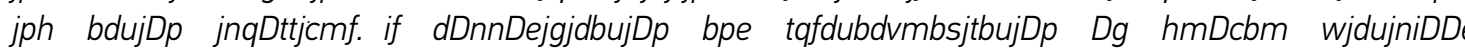

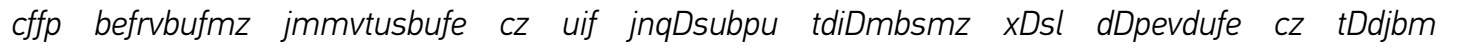
$\dot{E}<E ́ E><L B N>-K M$ ?? > B B @

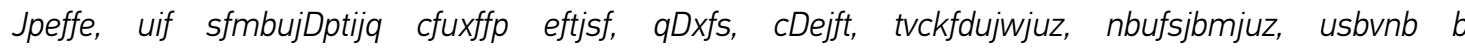

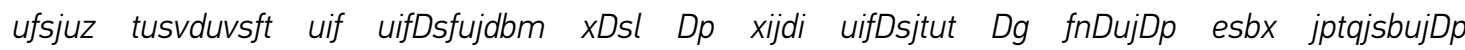

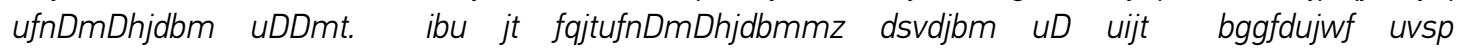

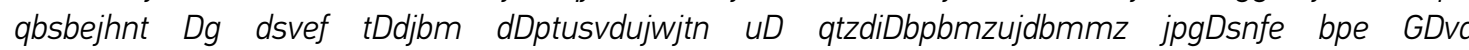

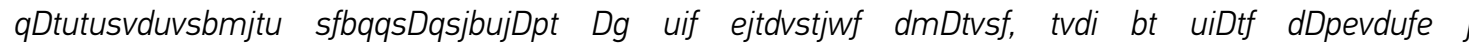

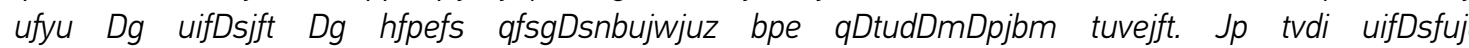

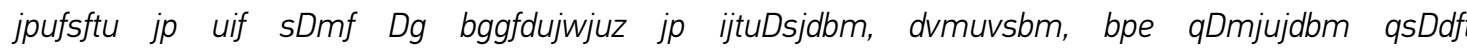

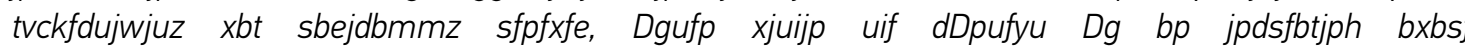

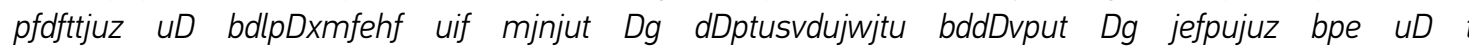

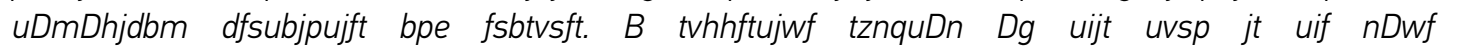

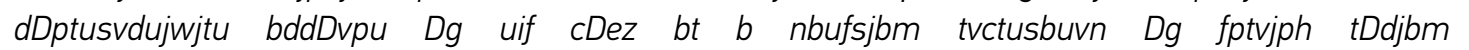

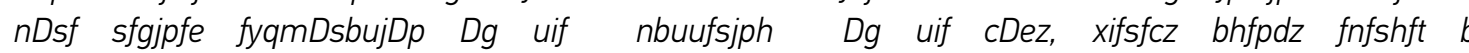
?

\section{\#FZPOE UIFFNPUJPODPHOJUJPOEJDIPUPNZ IJTUPSJDJTJOHJOUJNBUFTJUFTPG DPM POJBM BOE OB UJPOBM JTU H PW FSOBO}

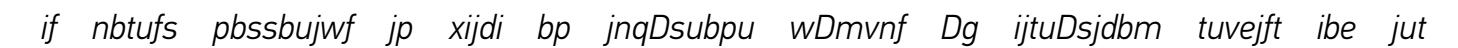
$J><>G L E Q \rightarrow G: E Q K>=\rightarrow G=-<A: E E A$ G

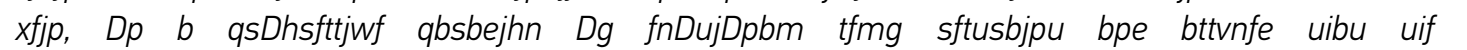

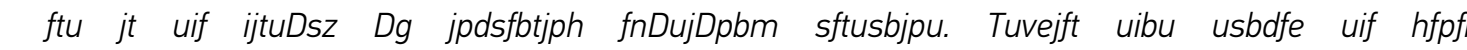

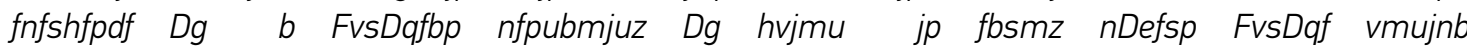

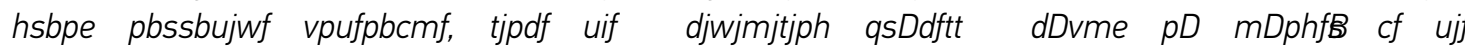

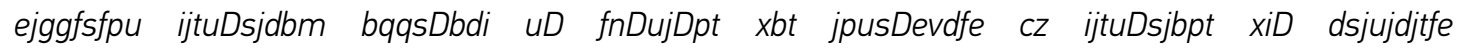

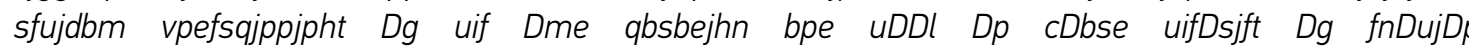

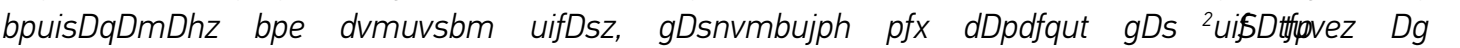

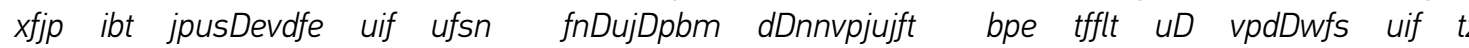

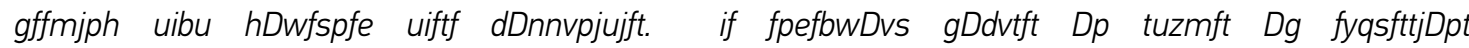

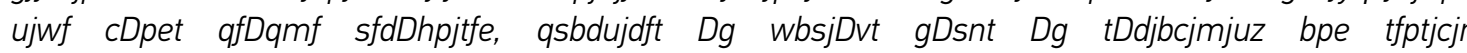

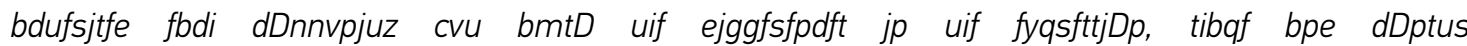

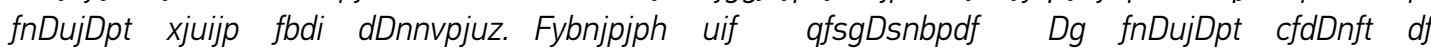




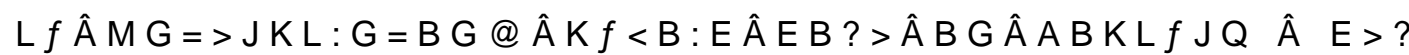

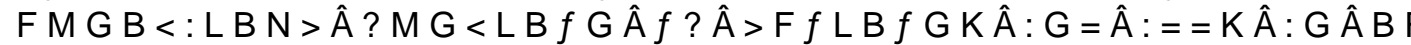

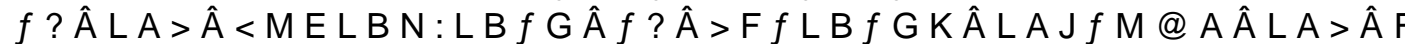

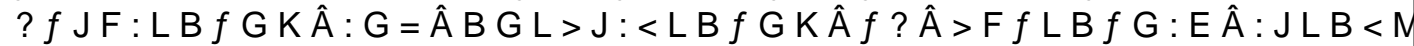

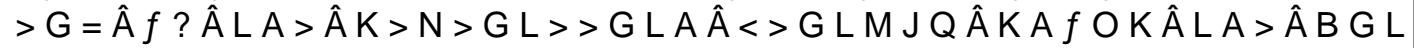
$H E ́ B L B<K \rightarrow G=\lfloor A>B J-M G=>J E Q B G @+H \dot{\theta}>>>>E: L B E ́ K A B H K$

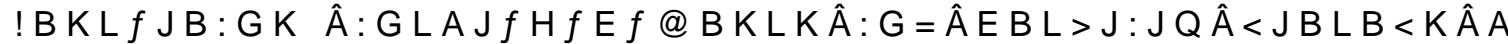

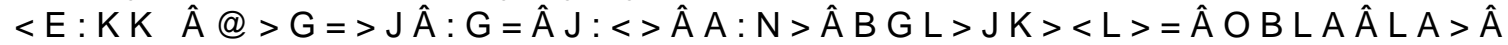

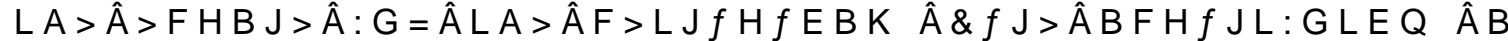

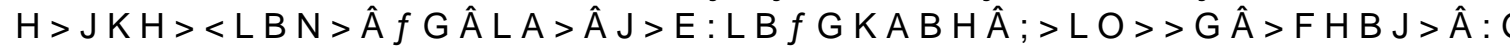

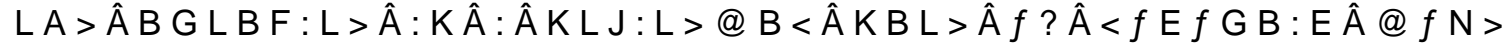

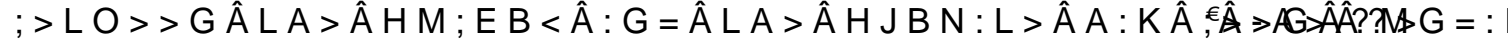

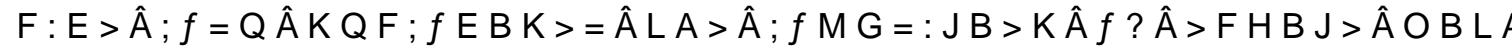

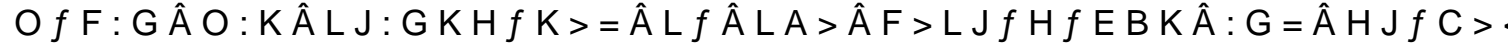

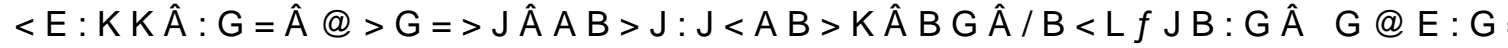

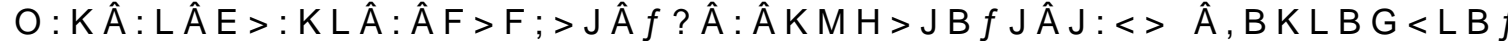

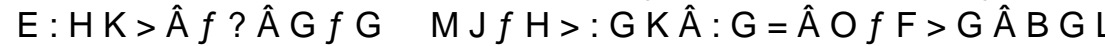

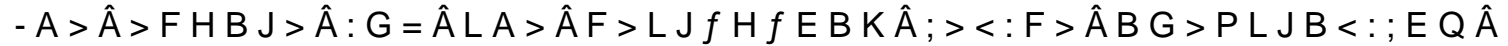

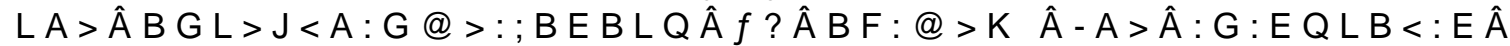

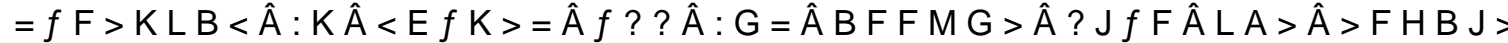
OÉDŁAJ们@A ŁA > $\rightarrow F H B J>$

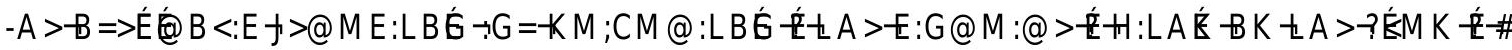

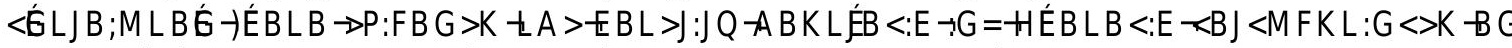

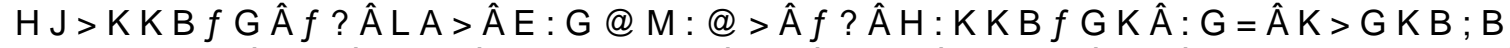

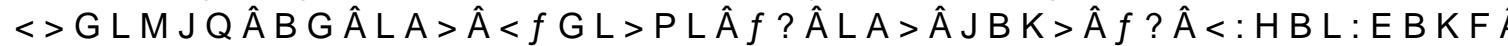

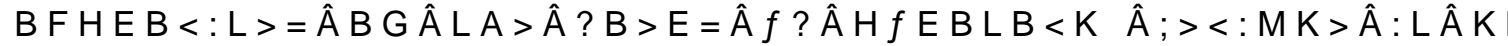

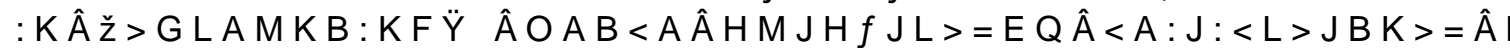

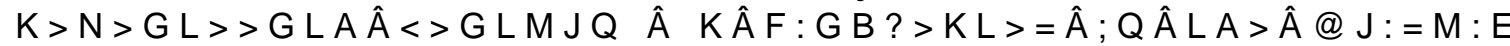

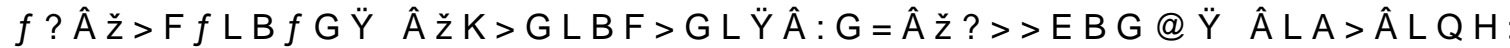
$:-K M ; E B F>-\neg B @ M J: L B N>E: G @ M: @>-0: K\lfloor A M K-K M H>\mid K>=>=\rightarrow Q \rightarrow-G>0 \dashv Q H>\dot{t}-A M F: G-K E$

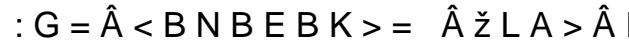

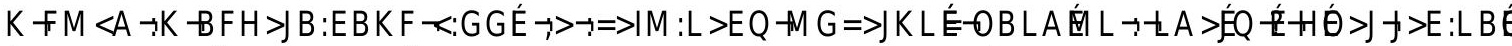

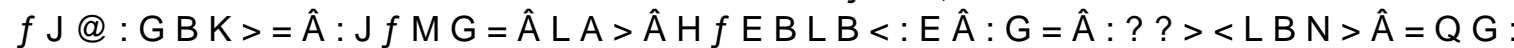

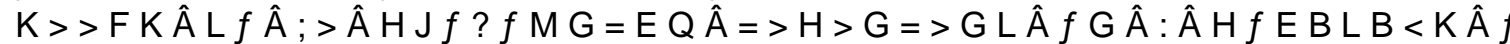

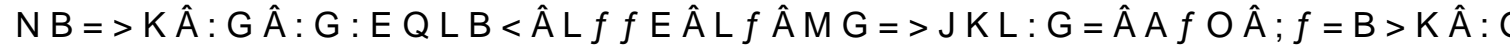

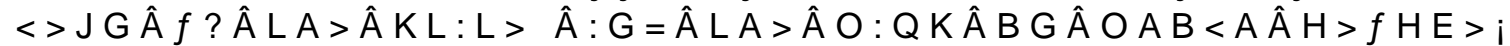

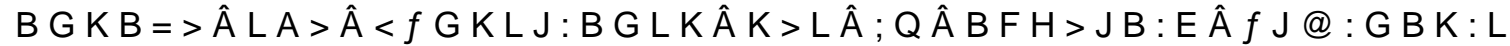

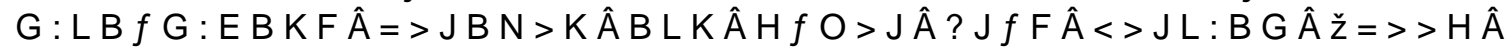

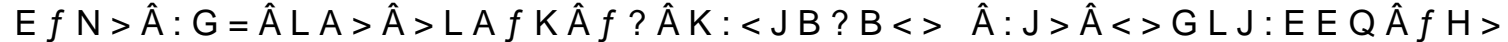

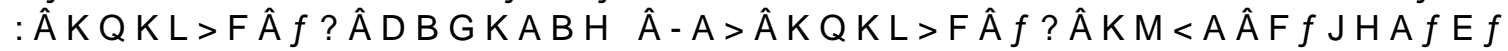


$\mathrm{G}: \mathrm{LBE} \rightarrow \mathrm{K} \rightarrow<\mathrm{E} F \mathrm{M}$ GB LQ

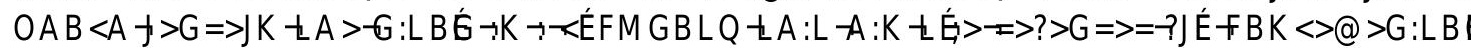

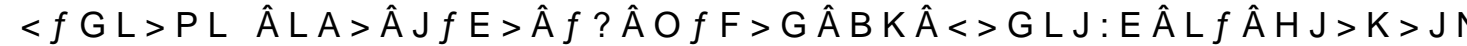

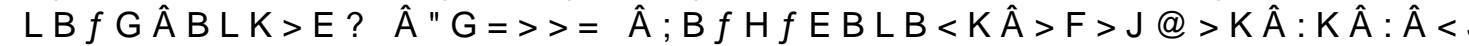

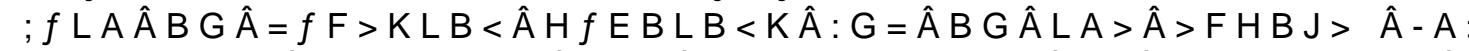

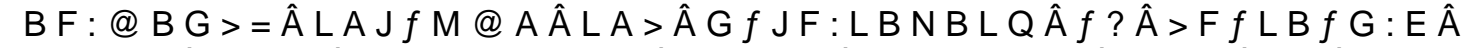

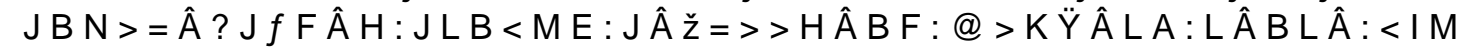
$L B>K-B G L A>G B G>L>>G L A \&>G L M J Q \neg$

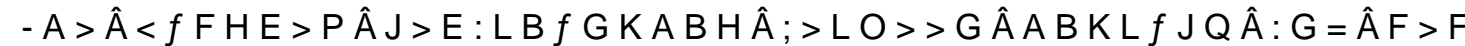

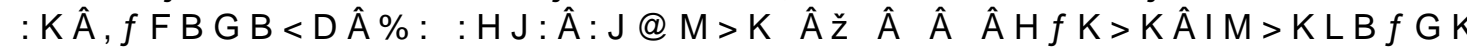

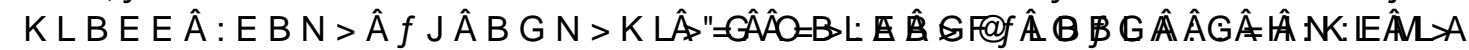

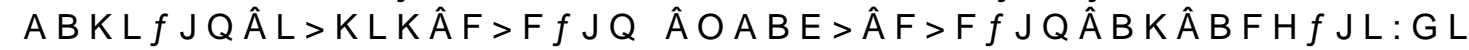

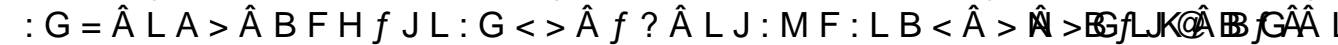

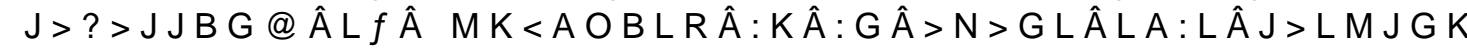
$L J: G K F B K K B$ É 壬

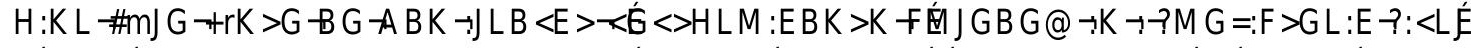

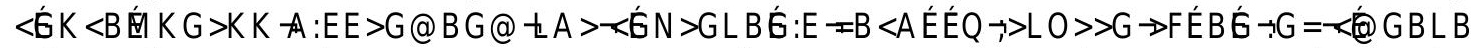

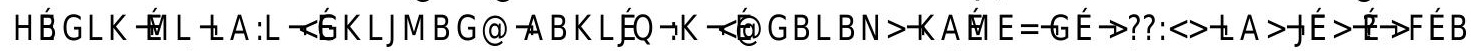

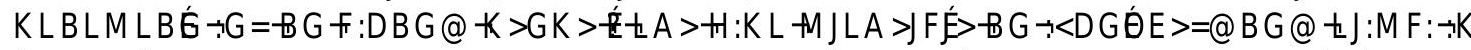

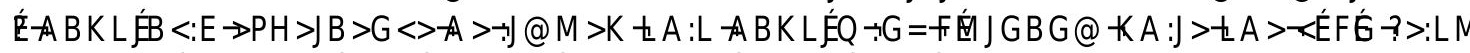

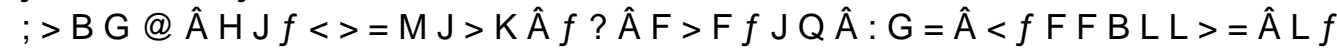

\section{*OUFSTFDUJPOT PG UIFEJTDVSTJWFB OE UIFQTZDIJD JOTPDJBM B OEIJTUPSJDBM QSPDFTTFT PG TV CKFDUJWBUJPO}

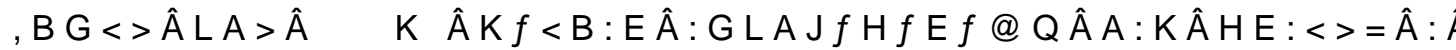

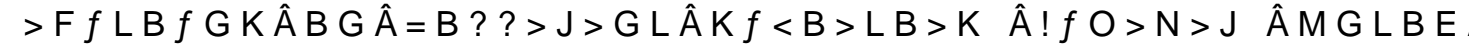

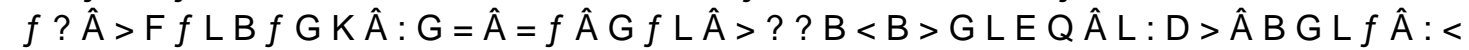

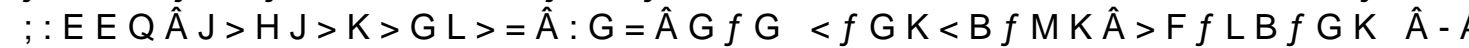
?B@MJ:LBÉ

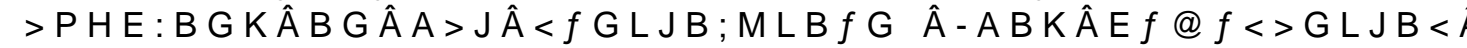

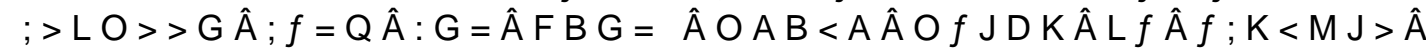

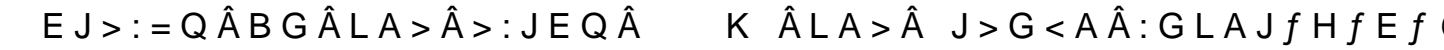

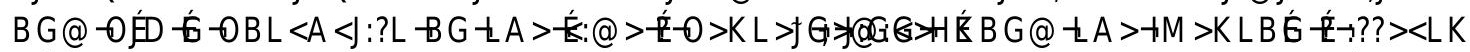

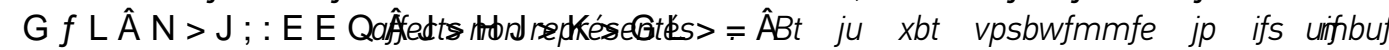

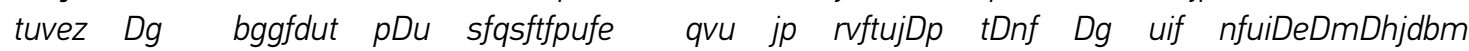

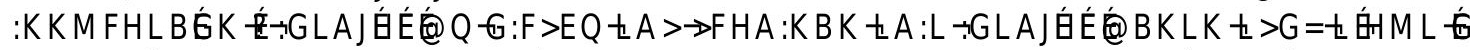

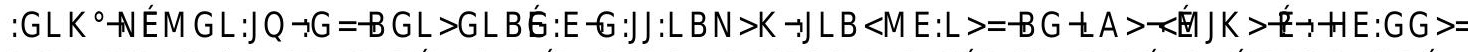

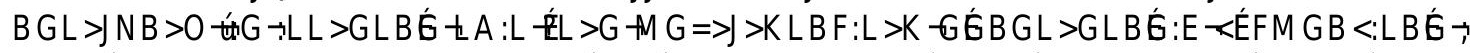

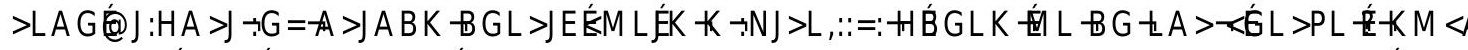

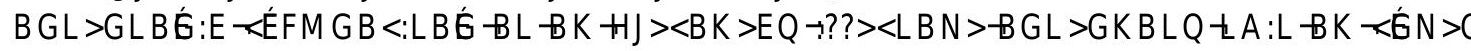




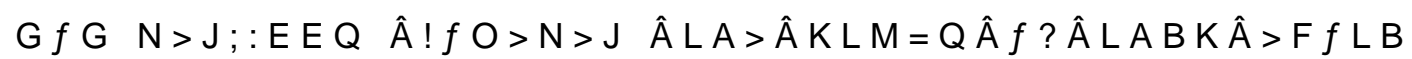

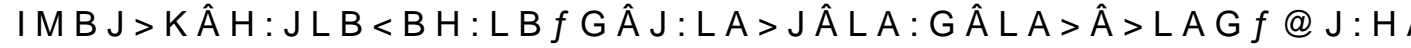

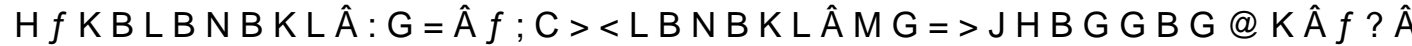

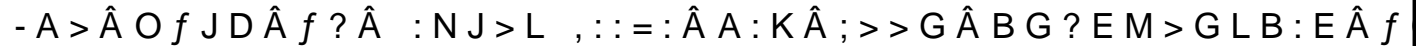

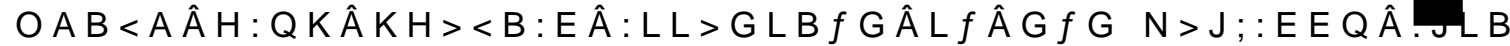
$<>H L B$ ÉK $\rightarrow A>K>\rightarrow G L A J$ 白囟 $B<: E-K L M=B>K \rightarrow P: F B G>E: G @ M: @>B L K>E$ ? $\rightarrow K \rightarrow-<$ 白 $>\dot{E}>: E-K>G K N$

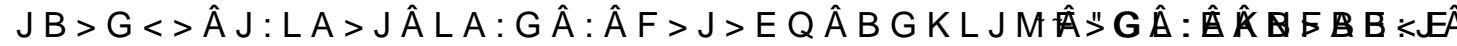

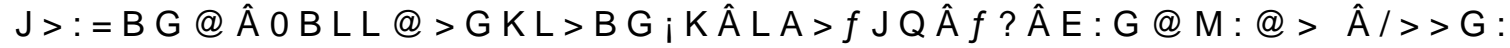

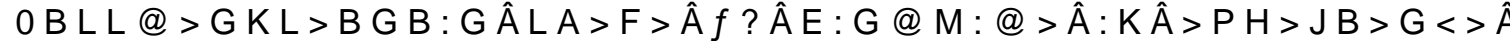

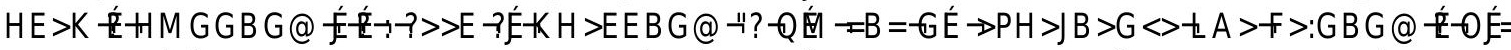

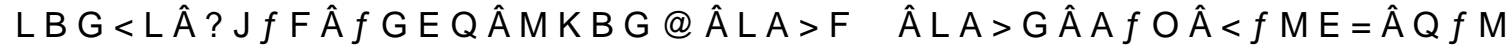

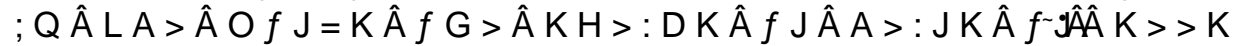

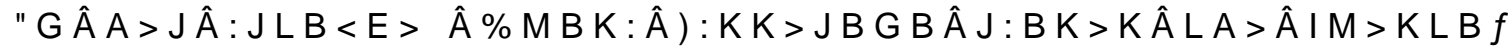

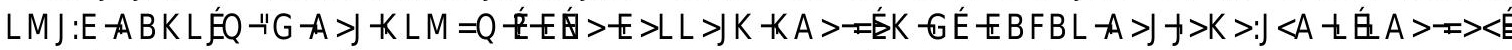

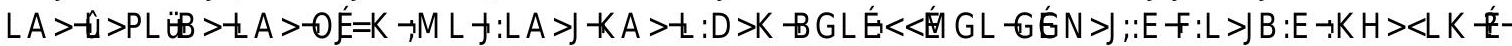

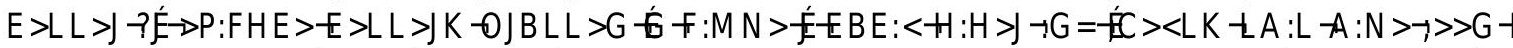

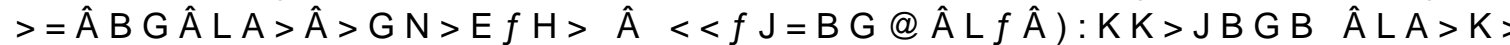

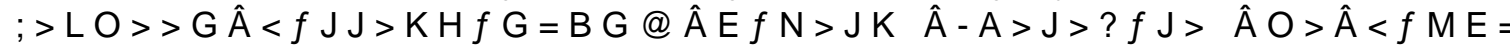

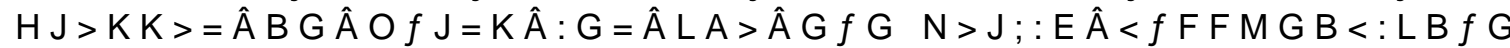

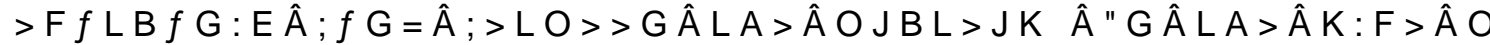

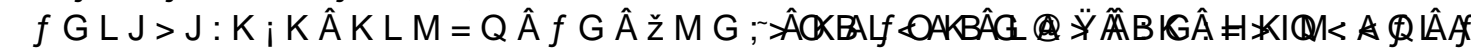

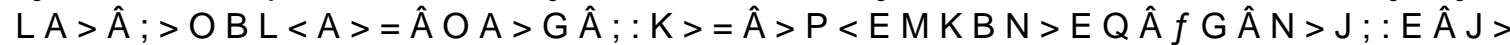

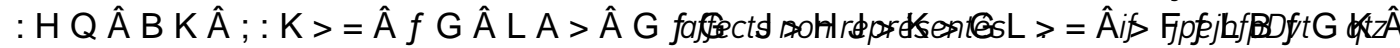

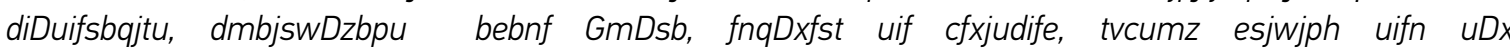

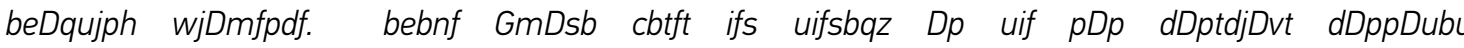

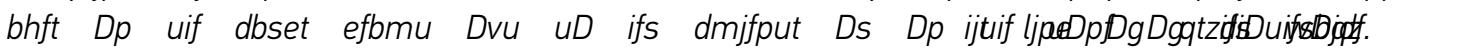

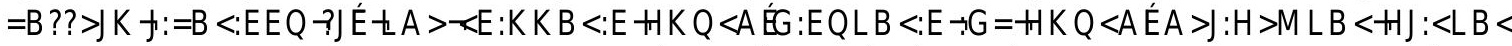

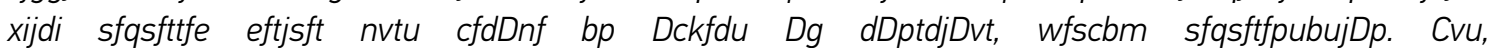

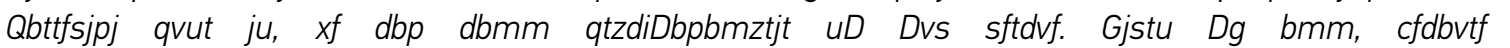

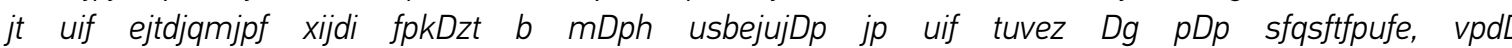
$?><L K \rightarrow G=-K><\dot{G}=E Q \rightarrow><: M K>-t A>1>\rightarrow$ l $>+H K Q<A$ É :EQ $L B<: E-K L M=B>K\lfloor A: L+M G K>L L E>-t A$ E囟 $E>G L J B<+H K Q<A E$ É:EQLB<:E HJ: $<L B<>\neg$

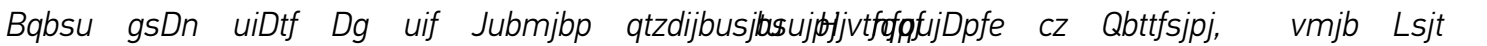

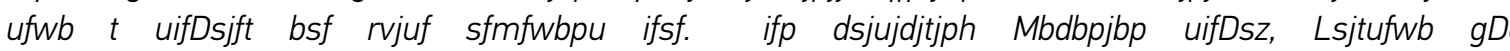

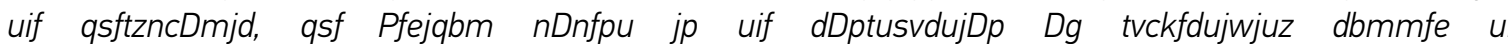

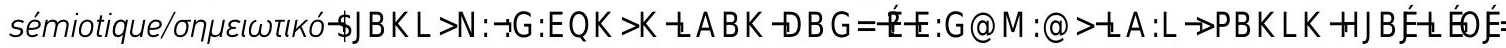

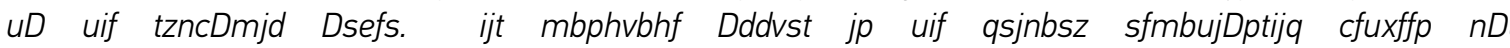

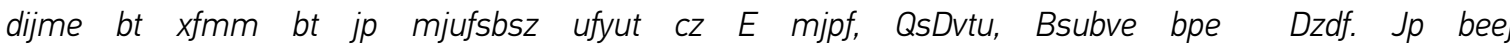

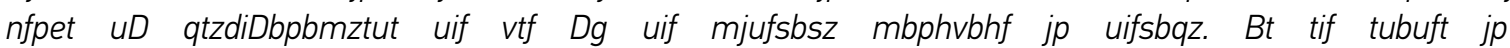

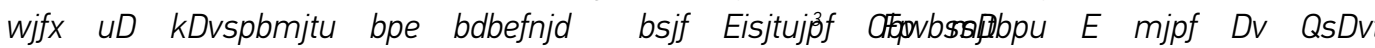

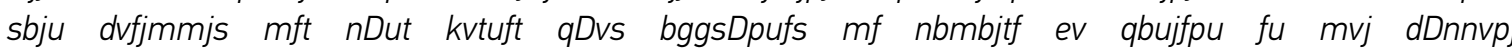




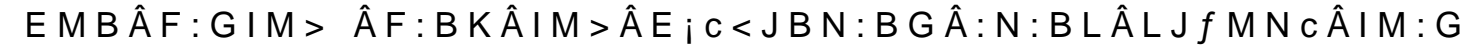

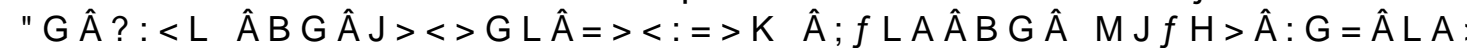

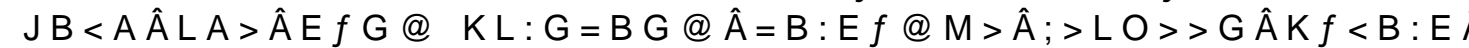

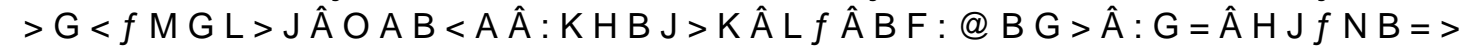

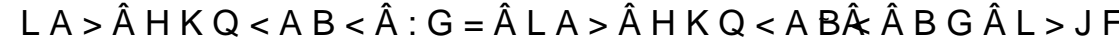

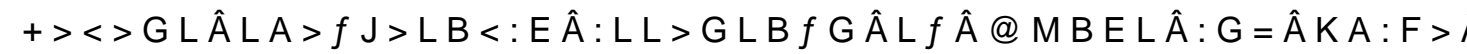

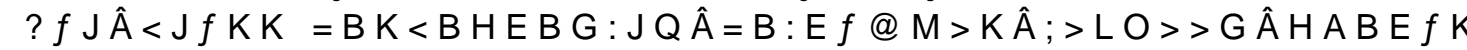

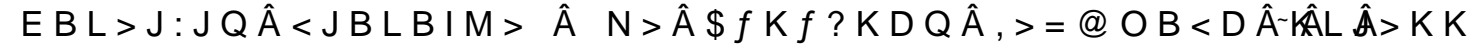

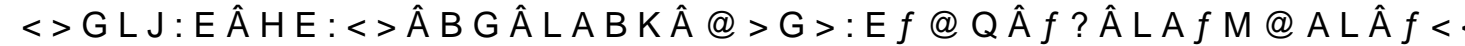

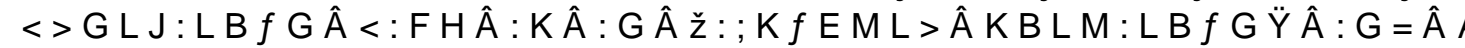

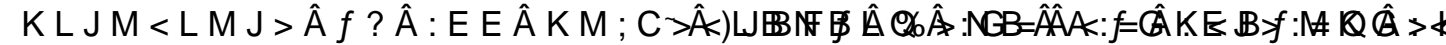

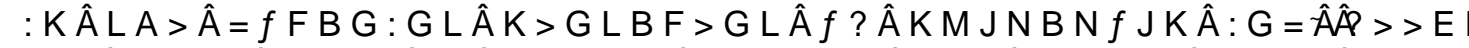

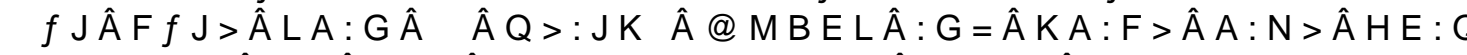
$B G F: L>K \quad B G\lfloor A><\dot{E}<>G L J: L B E<: F H \neg$

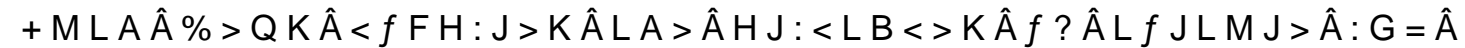

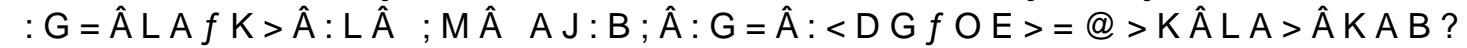

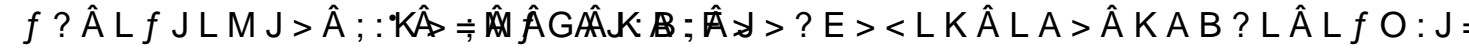

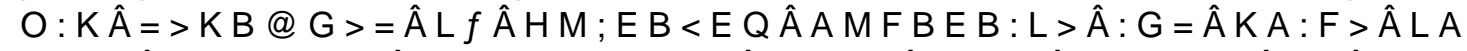

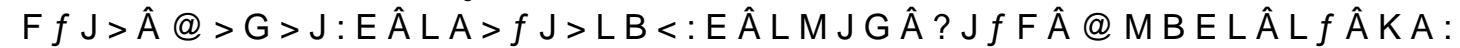

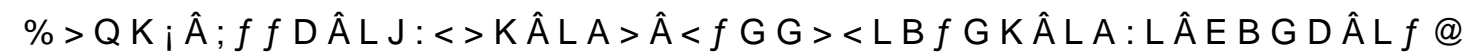

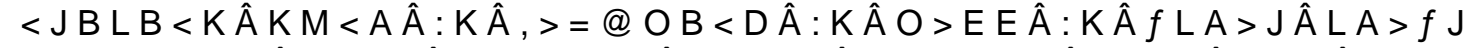

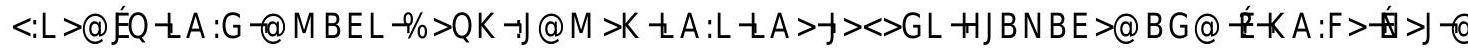

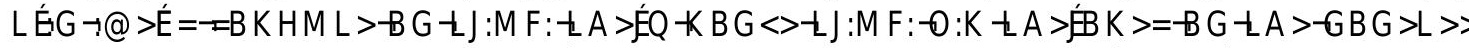

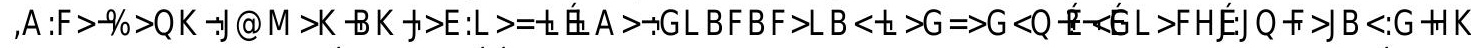

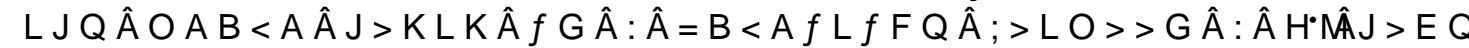

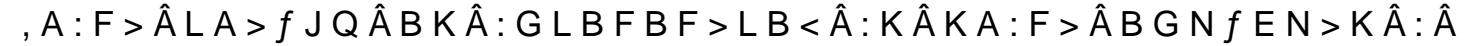

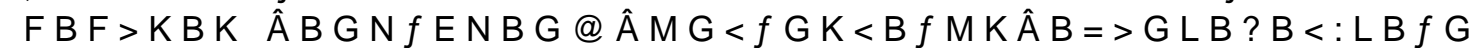

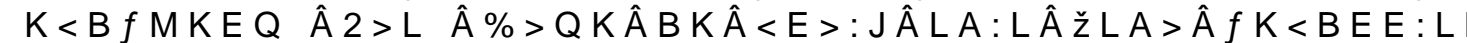

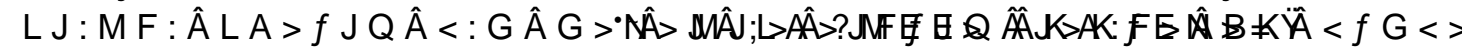

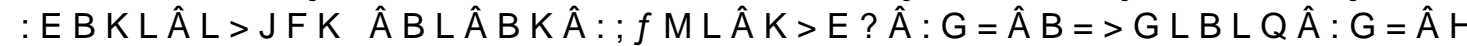

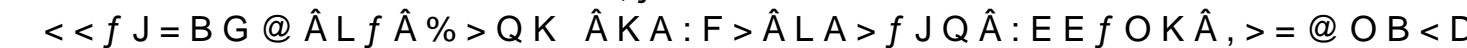

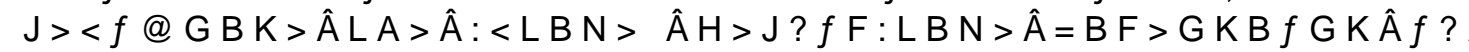

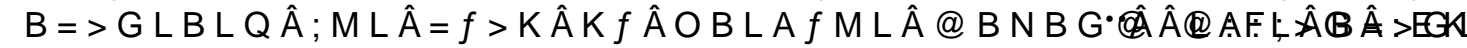

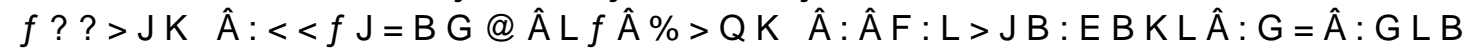

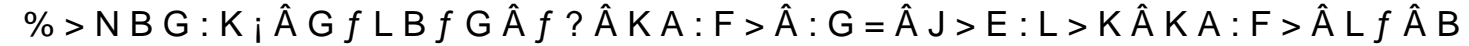

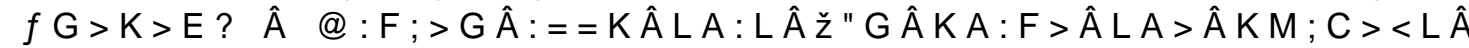

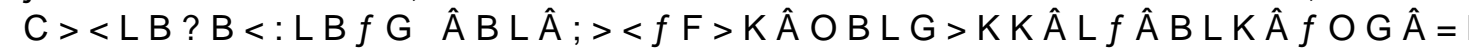

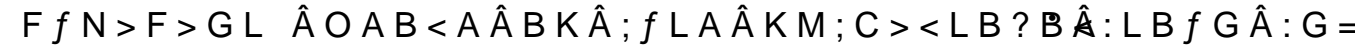

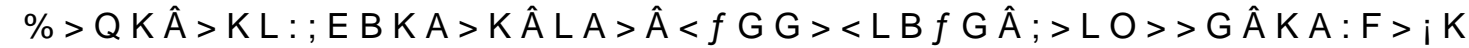

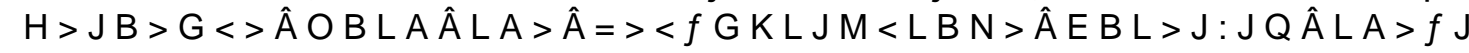




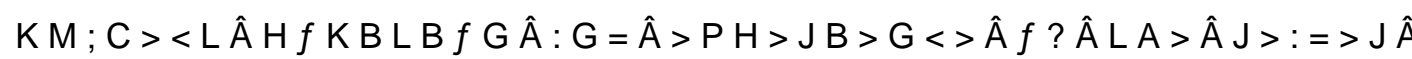

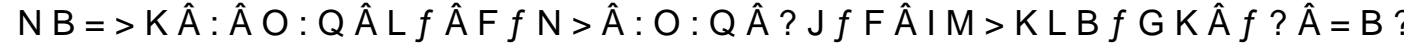

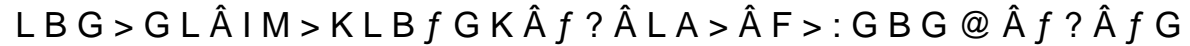

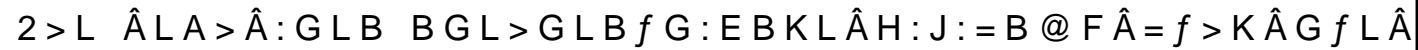

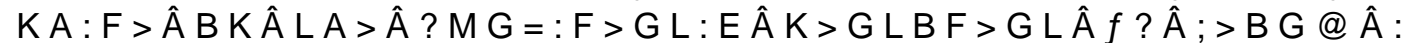
$K M ; C><L>=\div G=\rightarrow>B G @ K \dot{\theta}>>>B @ G \dot{\mathbb{4}} \oplus B L G>K K B G @ B L K \oplus G=>K M ; C><L B ? B<: L B E-\# M=B L A+M L$

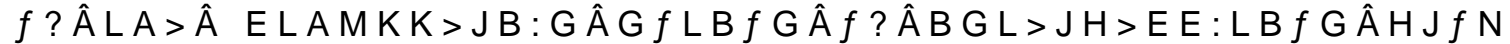

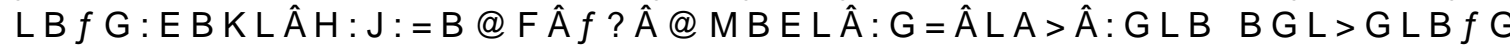

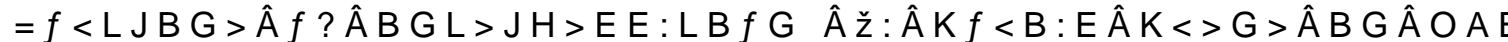

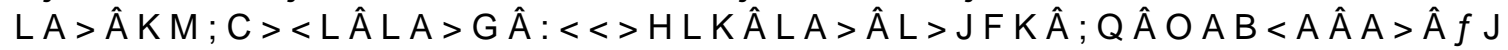

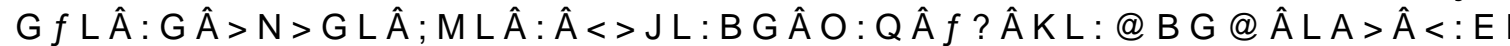

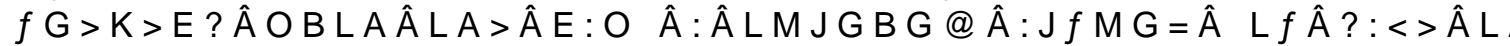

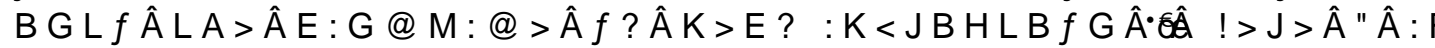

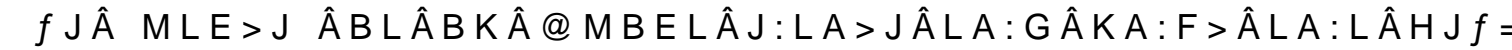

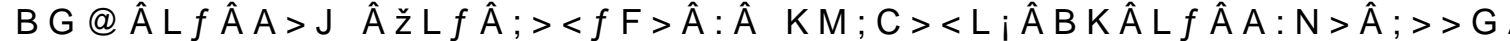

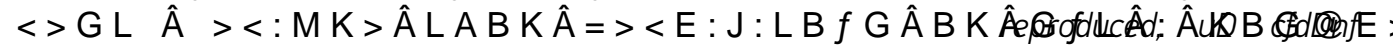

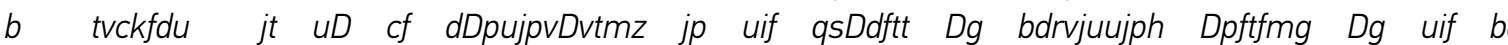

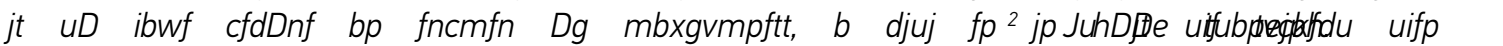
$L A: L+D>K$ B $=>E$ 囟 $Q$ -

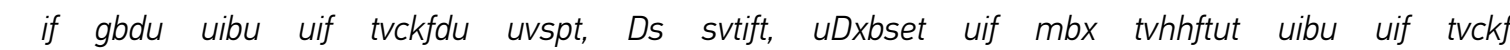

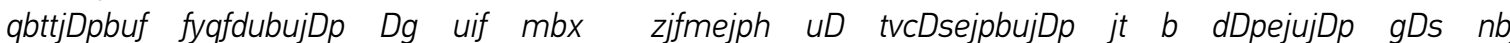

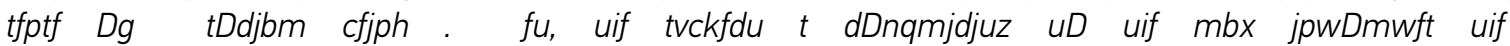

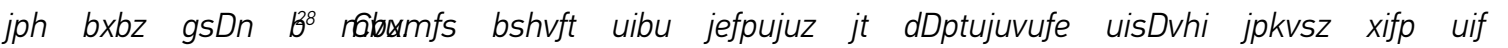

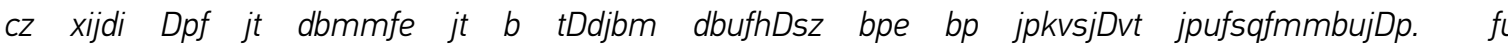

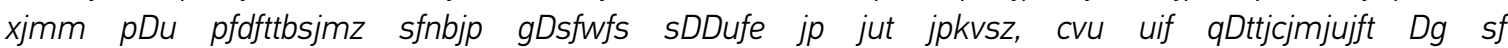

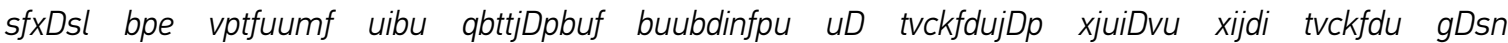

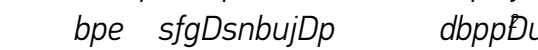

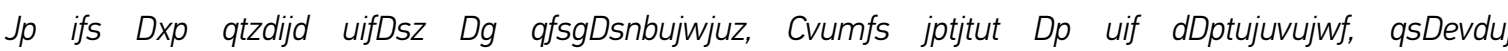

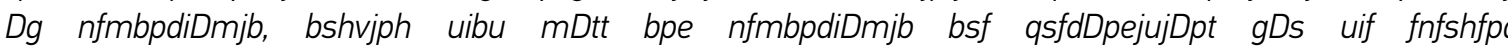

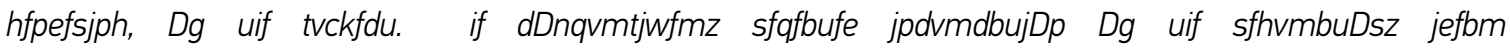

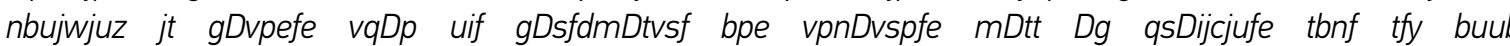

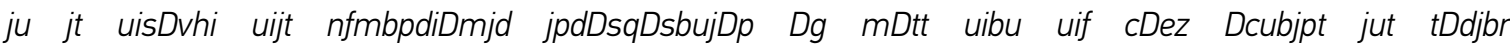

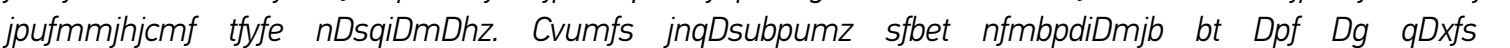

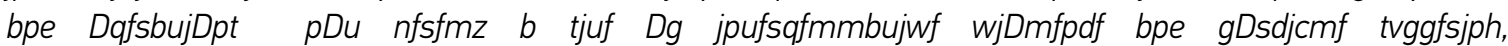

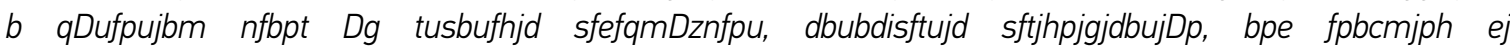

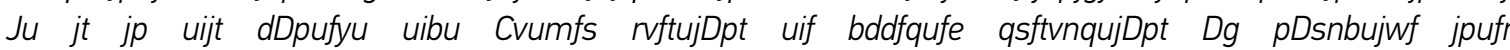

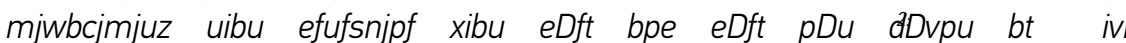

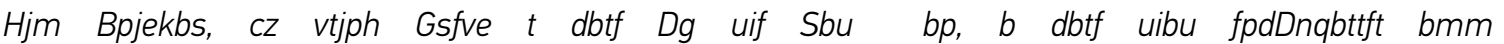

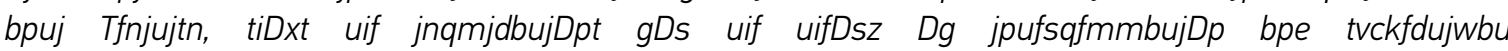




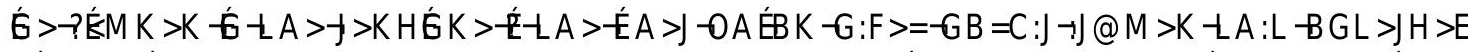

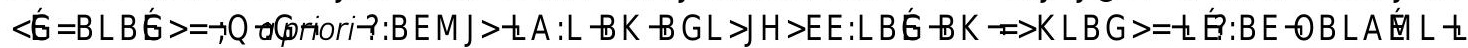

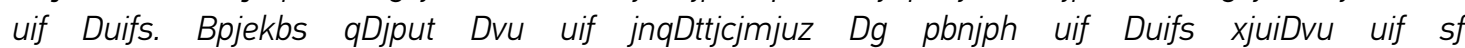

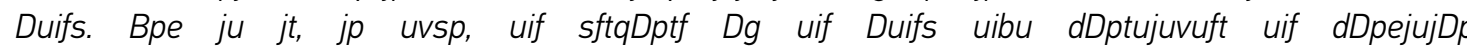

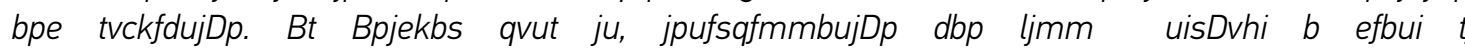
$H J E ́ M<>B=>G L B$ ?B $<: L B E ́-O B L A$ É $L \dashv>K H E K>-G=-0 A: L-A: H H>G K-B$ ? $-L A>-B G>N B L: ; B E B L$

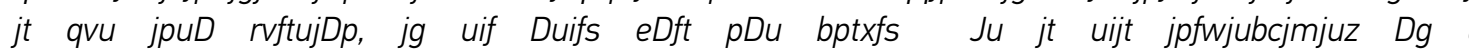

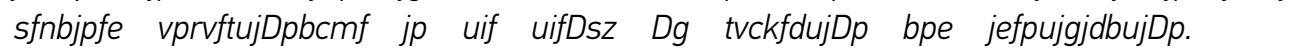

\section{\$PODM VTJPO}

$-A B K-B \Rightarrow>>: G @ B G @ \rightarrow G=B G K B @ A L ? M E \rightarrow G: E Q K B K \leftarrow \dot{E}: L>=-\cap B L A B G \pitchfork A>B G L>E E E D B G @-$

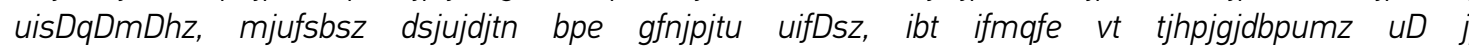

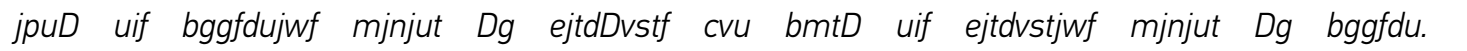

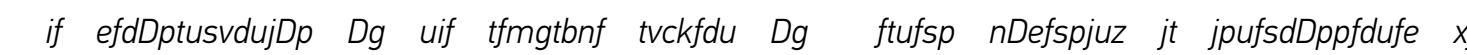

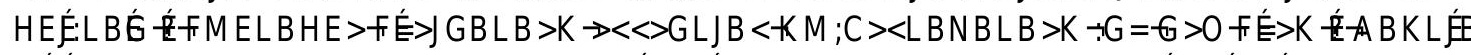

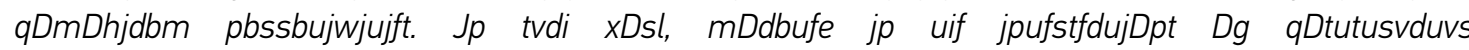
$B G K H B J>=\rightarrow G L B>K K>G L B: E B K L \rightarrow G=-\%:<: G B: G+H K Q<A$ É :EQ LB $<-7\}: F>0$ ÉDK $\downarrow A>-K M ; C><$

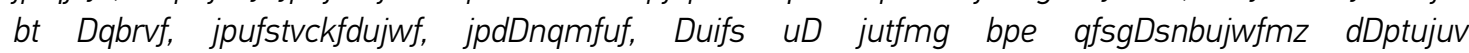

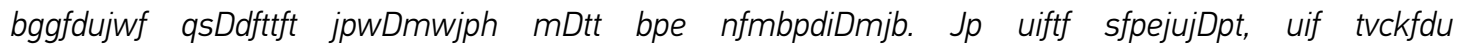
$: K \rightarrow+F>E: G<A E ́ B<\rightarrow @>G L\lfloor A: L \rightarrow G @: @>K-B G=B K<M J K B N>\rightarrow G=\rightarrow ? ?><L B N>+H J \dot{E}>K K>K$ t́t $K B=>+H \dot{\theta}>J-K L J M<L M J>K \neg$

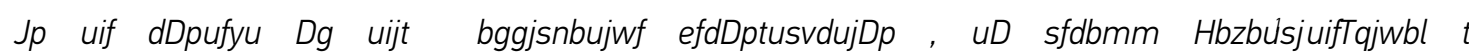

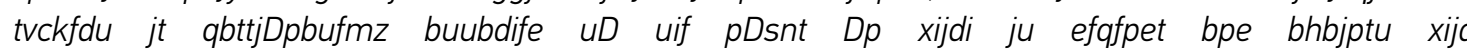

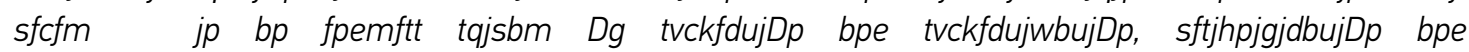

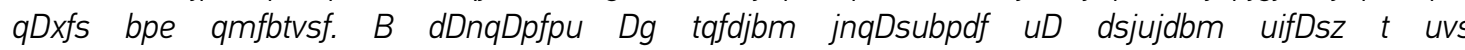

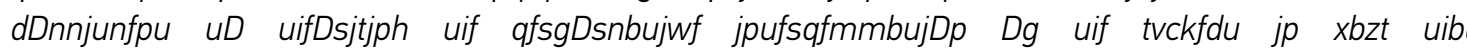

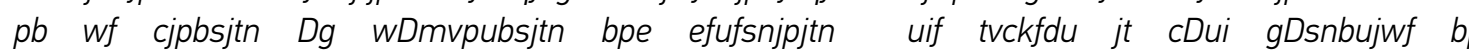

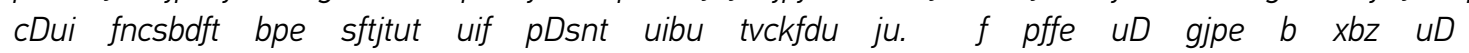
$\dot{E}>-K B=>-\dot{\theta}>\lfloor A>t \dot{E} A>J \neg$

Athena Athanasiou
Pothiti Hantzaroula
Kostas Yannakopoulos 


\section{NOTES}

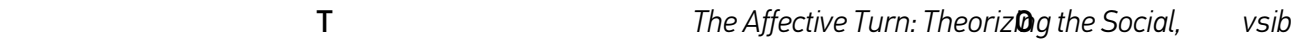
(G) D > ᄀ) $\sim \neg$

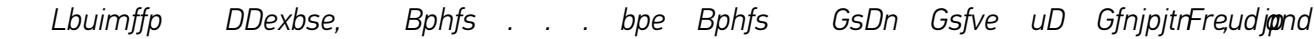

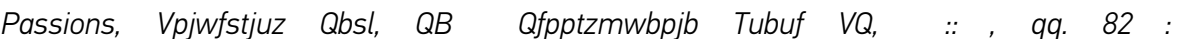

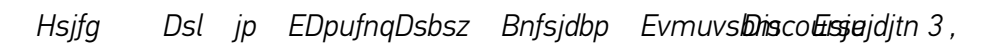

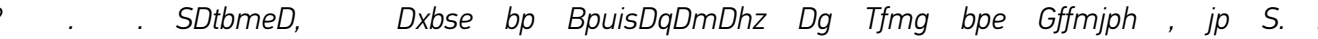
Culture Theory: Essays on Mind, Self, and Emotion $\neg F$; $B=@>\neg F ; j B=@>\neg) \neg r+1 \% M L R \rightarrow G=\neg$

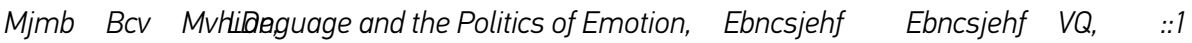

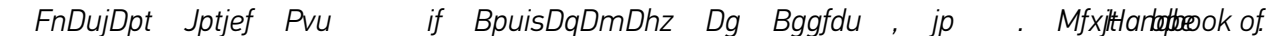

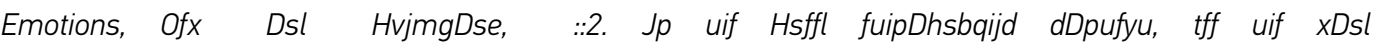
):H:L:PB:J $<A B K \rightarrow G=-i:=B: \neg>J>F>L: D B K$

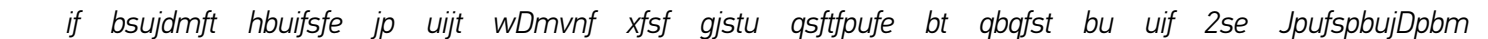

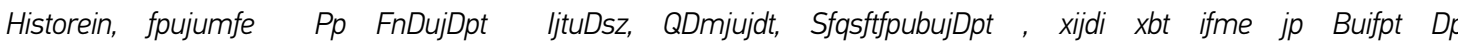

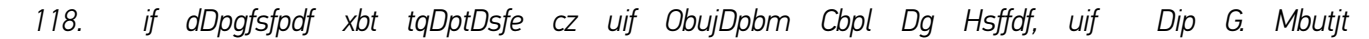

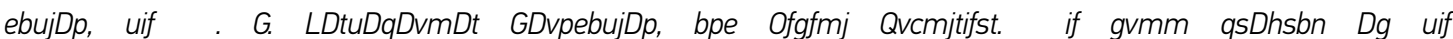

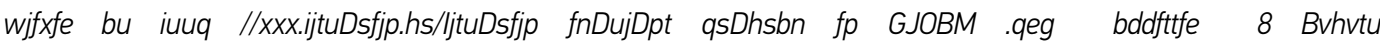

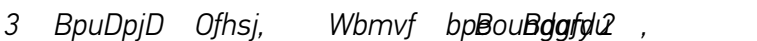

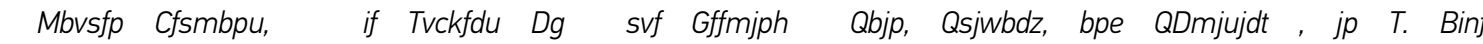

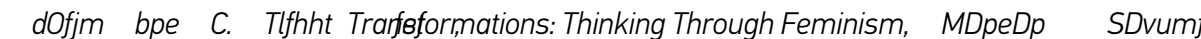

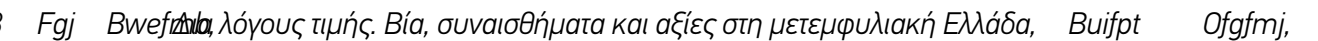

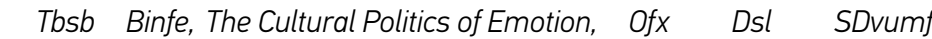

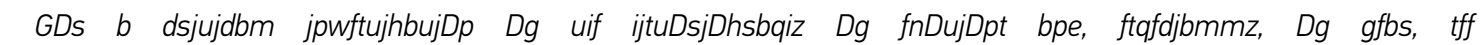

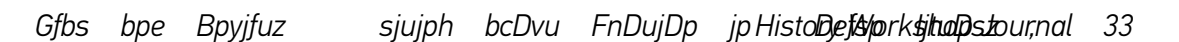

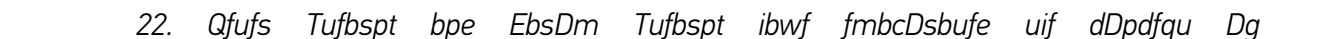

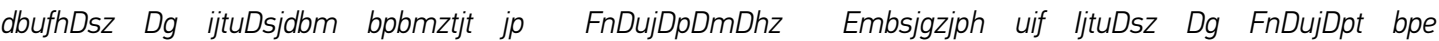

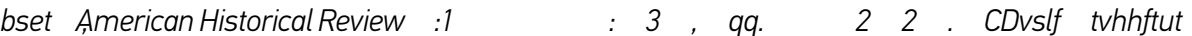

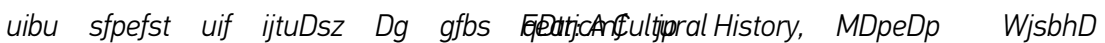

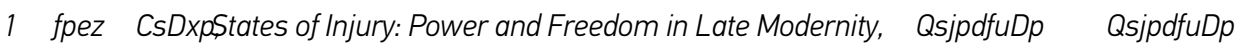

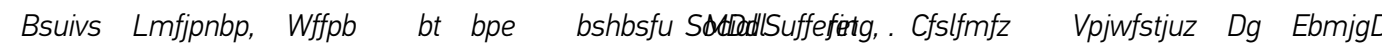
) $)>K K \rightarrow$

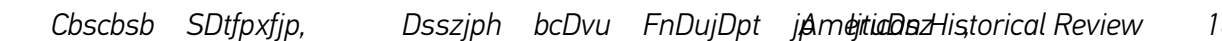
$\mathrm{HH} \rightarrow \ddot{\mathrm{A}}$

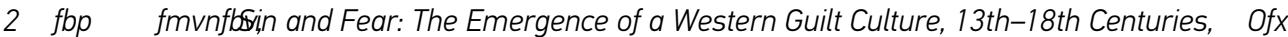

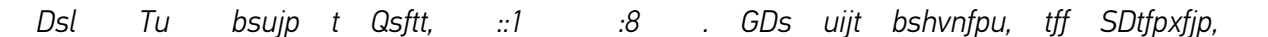

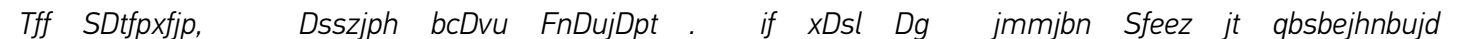

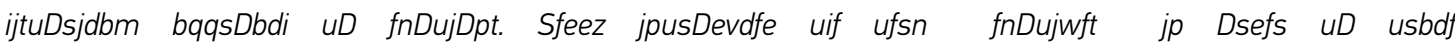

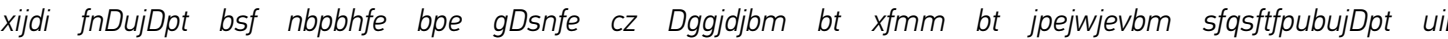
$N$ :J $B$ Él $K \neg$ ᄀ $L B<M E: L B E ́ K \neg$

$\ddot{A} \neg " ; B=+H H \sim \ddot{A}$ 


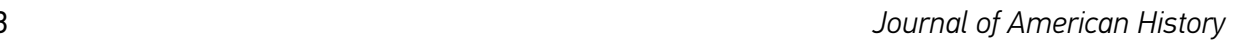
$\sim H H$ 世́

$\neg \mathrm{GG}-9: \mathrm{MJ}: \neg \mathrm{LE}$ E > Race and the Education of Desire: Foucault's History of Sexuality and the Colonial Order

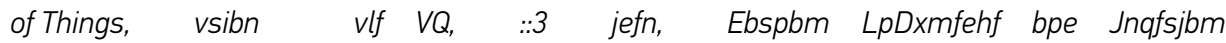

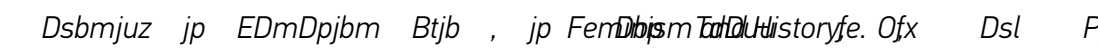

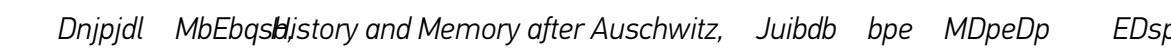
$\neg " ; B=$

$\sim \neg \mathrm{BÉ} @ \mathrm{BE} @: \mathrm{F} ;>\mathrm{G} \neg$ Remnants of Auschwitz: The Witness and the Archive $-\mathrm{L}]: \mathrm{GKE}-\{\mathrm{G} \mathrm{B}>\mathrm{E} \dashv>\mathrm{EE}>\mathrm{J}+\mathrm{R}>\mathrm{G} \neg$

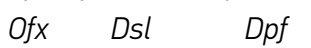

$\sim \neg \#: G G>\rightarrow N J>L \neg::=: \neg$ Les mots, la mort, les sorts $\rightarrow: J B K \neg: E E B F: J=\neg$

$\sim \sim \#>: G G>\rightarrow N J>L \neg::=:$ 过 $>\rightarrow ? ? ?><L$ c c̈̈Gradhiva $-H H$ फ́

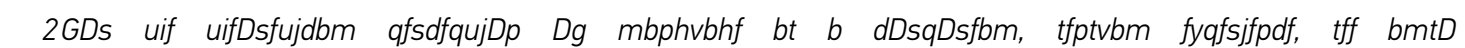

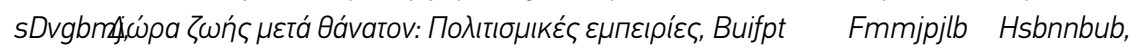

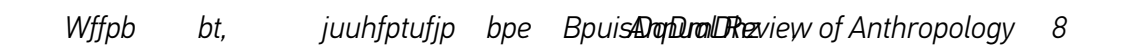

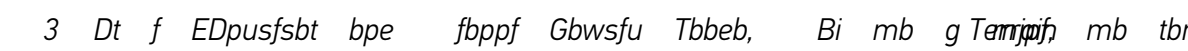

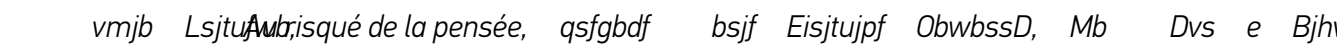

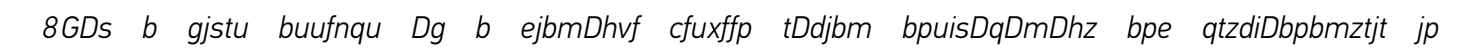

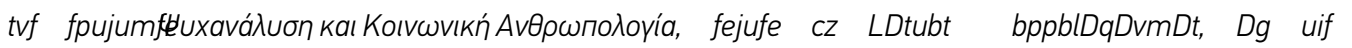

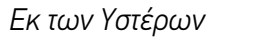

$\sim \neg N>$ >É ÉK DB ᄀ>=@ OB বDuaching Feeling: Affect, Pedagogy, Performativity

$\sim \neg @: F ;>G \neg$ Remnants of Auschwitz+H $\sim$

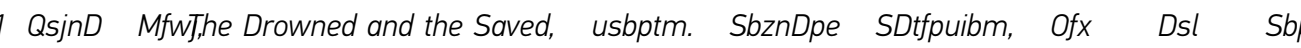
$\neg$

$\neg+\mathrm{M} \mathrm{LA}-\mathrm{Q} \%>$ Q

$\sim \neg "$; $=$ =H

$\neg " ; B=+H \neg$

$\neg " ; B=+H-\ddot{A}$

$\ddot{\mathrm{A}} \neg @: \mathrm{F} ;>\mathrm{G} \neg$ Remnants of Auschwitz+H

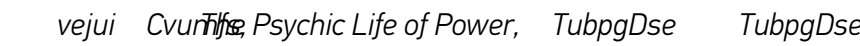

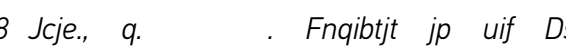

$\neg " ; B=H \neg$

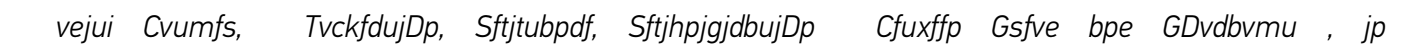

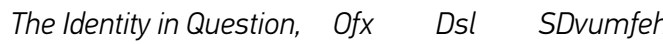

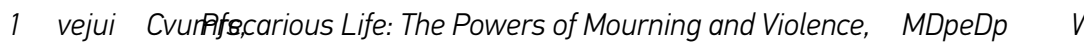

$\neg: Q: L J B-A: D J: N E ́ \mid$

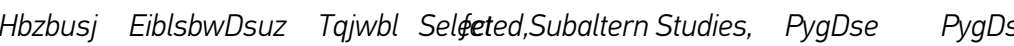

